\section{Richtlinie der Bundesärztekammer zur Qualitätssicherung laboratoriumsmedizinischer Untersuchungen}

\author{
G. Schumann \\ Hannover, Deutschland
}

\section{Synonym(e) RiLiBÄK}

Definition Gesetzlich vorgeschriebene Richtlinie zur Qualitätssicherung.

Beschreibung Die RiLiBÄK besteht aus einem allgemeinen Teil A, in dem ,grundlegende Forderungen an die Qualitätssicherung labormedizinischer Untersuchungen" gestellt werden. Dieser Teil ist sehr stark an ISO 15189 angelehnt. In den mehreren Teilen B1-B5 sind spezielle Anforderungen an die Qualitätskontrolle für viele in einem medizinischen Laboratorium angebotene Analysenverfahren festgelegt. Ein Teil $\mathrm{C}$ beschreibt Aufgaben und personelle Zusammensetzung eines Beirats. Die Teile D befassen sich mit spezifischen Fachgruppen für die Teile B. Die Teile E regeln die Bedingungen für B-spezifische Ringversuche. So gibt es beispielsweise für die quantitativen Laboratoriumsuntersuchungen B1 die speziellen Teile D1 und E1. In der Richtlinie werden Mindestanforderungen an die Qualitätssicherung, insbesondere an die Messgenauigkeit von quantitativen laboratoriumsmedizinischen Analysen festgelegt.

Mithilfe von Fehlergrenzen (zulässige maximale Messabweichungen) erfolgt eine Bewertung der Ergebnisse der internen und externen Qualitätssicherung. Die externe Qualitätssicherung besteht in der Teilnahme an einem $>$ Ringversuch. Die Einhaltung der Richtlinie ist für alle medizinischen Laboratorien, die der Patientenversorgung dienen, aufgrund der Medizinprodukte-Betreiberverordnung vorgeschrieben.

\section{Literatur}

Richtlinie der Bundesärztekammer zur Qualitätssicherung laboratoriumsmedizinischer Untersuchungen (2014) Dtsch Ärztebl 111: A1583-A1618 